\title{
Complex interactions between the replicating oncolytic effect and the enzyme/prodrug effect of vaccinia- mediated tumor regression
}

\author{
JA McCart, M Puhlmann, J Lee, Y Hu, SK Libutti, HR Alexander and DL Bartlett \\ Surgery Branch, National Cancer Institute, National Institutes of Health, Bethesda, MD, USA
}

\begin{abstract}
Replicating viruses for cancer gene therapy have beneficial antitumor effects, however, in the setting of an enzyme/prodrug system, the interactions between these viruses and the activated agents are complex. A replicating vaccinia virus expressing the cytosine deaminase gene (VVCD), which converts the prodrug 5-FC into 5-FU, was characterized in vitro and in vivo for its antitumor effects and pathogenicity. Replicating VVCD ( $\pm 5-F C)$ at various MOIs was used to infect MC38 murine colon adenocarcinoma cells. At high MOls $(>0.1)$ virus alone was able to kill the majority (65-90\%) of cells by day 5 with no additional benefit from prodrug. At low MOls only the effect of prodrug is seen. Cell lysates demonstrated 300-fold reduced viral recovery from cells treated with both VVCD and 5-FC compared with
\end{abstract}

controls treated with virus alone. Nude mice bearing subcutaneous MC38 tumors were injected with VVCD (or control) and treated with 5FC or control. Mice injected with VVCD (with or without 5FC treatment) had smaller tumors than the controls, suggesting that replicating vaccinia alone is cytotoxic to tumors in vivo. The addition of 5-FC improved the antitumor response when a low dose of virus was injected into tumors. Also, compared with mice that received virus alone, those that received VVCD and 5FC had significantly prolonged survival from virus-mediated death. In conclusion, the addition of an enzyme/prodrug system to a replicating virus can improve the antitumor response and decrease viral pathogenicity. Gene Therapy (2000) 7, 1217-1223.

Keywords: replicating vaccinia virus; gene therapy; cytosine deaminase; enzyme/prodrug

\section{Introduction}

Tumor-directed gene therapy is limited by the inefficiency of current vectors such as liposomes, retroviruses and adenoviruses. Although viruses have been modified to allow tumor targeting and tumor-specific gene expression, ${ }^{1-4}$ transduction efficiencies and levels of gene expression remain low and limit the therapeutic effects. Replicating viruses have several advantages, including high levels of gene expression, increased transduction efficiencies through replication and infection of surrounding cells, and antitumor effects via virus-mediated cell death. ${ }^{5-8}$ Although the safety of replicating vectors has been questioned, 9 the development of tumor-specific replicating vectors should allow for viral replication within the tumor, but little systemic pathogenicity.

The inclusion of an enzyme/prodrug system with an oncolytic virus has several potential advantages. ${ }^{10-13}$ Diffusion of the converted prodrug into uninfected cells should lead to increased antitumor effects in vivo. Prodrug can be withheld until maximum viral replication and gene expression occur, allowing for maximal prodrug conversion. The use of converted prodrugs which inhibit DNA synthesis, may decrease viral DNA syn-

Correspondence: DL Bartlett, Surgery Branch, NCI, NIH, Building 10, Rm 2B16, 9000 Rockville Pike, Bethesda, MD, 20892, USA

Received 30 July 1999; accepted 27 April 2000 thesis, and limit replication. The prodrug may act to rescue the host from systemic viral pathogenicity.

Vaccinia virus has several advantages for use in tumordirected gene therapy. ${ }^{14,15}$ It is a large, cytoplasmic virus which does not require host machinery for DNA synthesis and up to $25 \mathrm{~kb}$ of foreign DNA can be inserted without the need for viral deletions. ${ }^{14,16}$ Vaccinia virus has an efficient replication cycle and infects most cell types. Previously, a thymidine kinase-deleted virus has been shown to have decreased pathogenicity compared with wild-type virus, with preserved replication in tumor cells. ${ }^{17}$ A thymidine kinase-deleted virus obtains thymidine triphosphate for DNA synthesis from the nucleotide pool only present in actively dividing cells. This leads to preferential viral replication in dividing cells, and hence the observed tumor specificity. Treatment with $10^{6}$ p.f.u. of vaccinia virus and the enzyme/prodrug systems cytosine deaminase (CD)/5-fluorocytosine (5-FC) or purine nucleoside phosphorylase (PNP)/6-methylpurine deoxyriboside (6-MPDR) led to long-term cures in a murine model of hepatic metastases. ${ }^{18,19}$

The purpose of this study was to examine the interactions between a replicating oncolytic virus and an enzyme/prodrug system with respect to antitumor effects and viral pathogenicity. We demonstrate dosedependent virus and enzyme/prodrug effects. Highdose, systemically delivered, replicating (thymidine kinase-deleted) vaccinia virus selectively replicates in a subcutaneous murine colon carcinoma compared with nor- 
mal tissues, leading to inhibition of tumor growth. Inclusion of the CD/5-FC system enhances the antitumor effect of low-dose vaccinia treatment, and decreases pathogenicity of high-dose vaccinia treatment.

\section{Results}

\section{In vitro cytotoxicity of vaccinia/CD/5-FC}

MTT assays were performed using a replicating virus in order to characterize the interaction between virusmediated cell death and enzyme/prodrug effect and to determine if the converted prodrug (5-FC $\rightarrow 5-\mathrm{FU})$ would inhibit viral replication. Replicating VVCD at an MOI of 1.0 resulted in $13 \%$ and $9 \%$ cell viability in MC38 cells by day 5 with or without the addition of prodrug respectively (Table 1). Similarly, an MOI of 0.1 resulted in $38 \%$ and $32 \%$ cell viability by day 5 with or without the addition of prodrug. An MOI of 0.01 plus $5-\mathrm{FC}$ ( $1 \mathrm{~mm}$ ) led to $61 \%$ cell viability by day 5 , illustrating a strong bystander effect for the CD/5-FC system. Without prodrug, virus alone caused minimal cytotoxicity by day 5 at this MOI.

In the presence of $1 \mathrm{~mm} 5 \mathrm{FC}$ viral replication was inhibited in a dose-dependent fashion from a six-fold decrease in viral recovery at an MOI of 0.001 to a 300fold decrease in recovery at an MOI of 1 . This suggests that inhibition of viral replication is dependent upon the amount of CD which converts the 5-FC into the active 5 -FU. The inclusion of a suicide gene in a recombinant, replicating vaccinia virus may allow for rescue from viral pathogenicity as well as the cytotoxic bystander effect.

\section{In vivo marker study}

Vaccinia virus expressing firefly luciferase (VVLuc) was injected into subcutaneous MC38 tumor-bearing nude mice to determine the optimal route of administration, timing of maximal gene expression and viral localization. $10^{7}$ p.f.u. of VVLuc was injected intravenously (i.v.), intraperitoneally (i.p.), or intratumorally (i.t.). Intraperitoneal administration resulted in high levels of gene expression in the tumor, which was 3 to 4 logs higher than the next highest tissue (ovary) (Figure 1a) and similar profiles were seen with i.v. and i.t. delivery (data not shown). Minimal luciferase expression was seen in the other organs tested. Gene expression in the tumor peaked on day 4 after i.v. delivery, and day 6 after i.p. and i.t. delivery, and remained high at all time points thereafter (Figure 1B).

Table 1 Percentage viability ( \pm s.d.) of MC38 cells 5 days after treatment with replicating vaccinia at various MOIs \pm 5 -FC

\begin{tabular}{lrrrc}
\hline MOI & 1 & 0.1 & 0.01 & 0.001 \\
\hline VVCD + 5FC & $12 \pm 0.9$ & $38 \pm 2$ & $61 \pm 8$ & $97 \pm 7$ \\
VVCD + HBSS & $9 \pm 1^{\mathrm{a}}$ & $32 \pm 1$ & $95 \pm 3$ & $104 \pm 4$ \\
5FC (no virus) & $95 \pm 2^{\mathrm{a}}$ & $107 \pm 2$ & $98 \pm 6$ & $94 \pm 0.5$ \\
\hline
\end{tabular}

MC38 cells were infected with replicating vaccinia at the MOIs listed above. The next day $1 \mathrm{~mm}$ 5-FC or HBSS was added. On day 5 MTT assays were performed and percent viability calculated. Each experiment was done in triplicate.

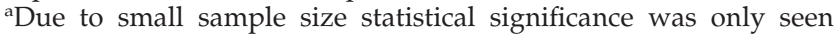
between these groups $(P<0.01)$.

\section{a}

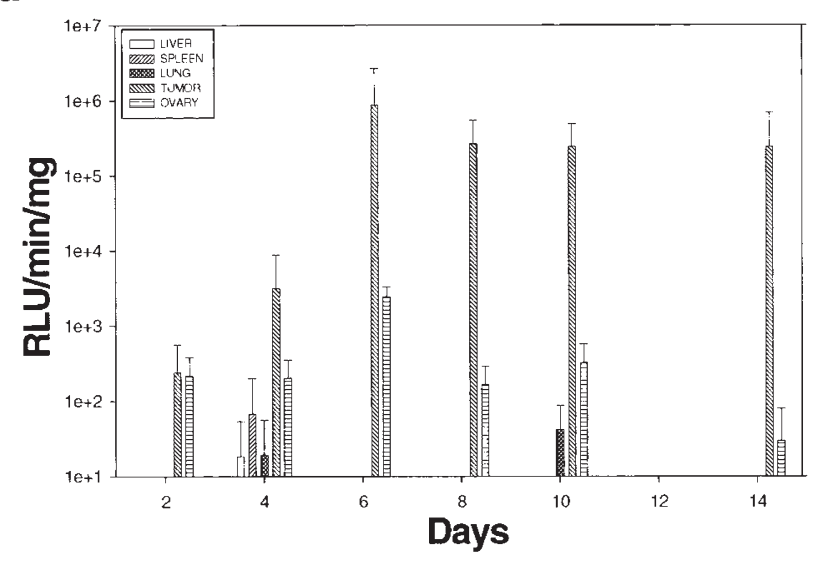

b

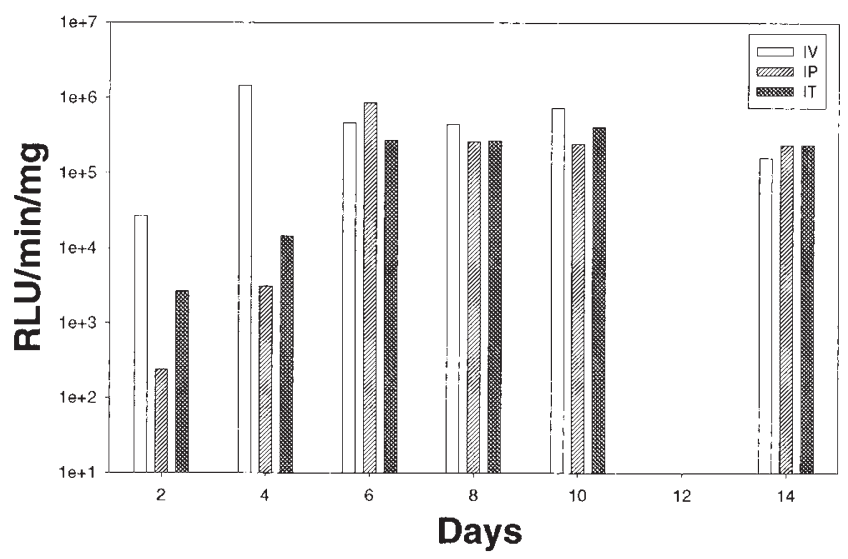

Figure 1 Luciferase levels (RLU/min/mg), 2 to 14 days after viral infection. (a) Luciferase levels in liver, spleen, lung, tumor and ovary after nude mice $(n=3)$ were infected i.p. with a luciferase expressing vaccinia virus (VVLuc). Tumor expression peaks on day 6 and remains high. Ovary, the second highest expressing organ, was 3 logs lower by day 6 $(P<0.01$ on days 6 and 14). Small bowel, heart, and skin were negative (data not shown). (b) Tumor luciferase levels when virus was given i.v., i.p., or i.t. By day 6 all routes of delivery were equivalent and levels remain elevated in tumor for the duration of the experiment.

\section{In vivo anti-tumor effect}

Nude mice with subcutaneous MC38 tumors (50 to $100 \mathrm{~mm}^{3}$ ) were treated with i.t. or i.p. replicating vaccinia virus expressing cytosine deaminase or control. 5-Fluorocytosine or control was given i.p. for 14 days starting on day 6 after viral delivery. $10^{7}$ p.f.u. of i.t. VVCD plus 5FC resulted in significant inhibition of tumor growth compared with controls ( $P<0.01$, Figure $2 \mathrm{a}$ ), with five of 10 mice having complete tumor regressions, and three long-term cures (two mice died at a later date from possible viral toxicity). Similar results (two of 10 long-term cures) were seen at $10^{8}$ p.f.u. of VVCD $+5-F C$ given i.t. (Figure 2b). Although there was inhibition of tumor growth at both doses of i.t. VVCD alone, there were no complete regressions and it did not reach statistical significance (Figure $2 a$ and $b$ ).

VVCD ( $10^{7}$ p.f.u.) given i.p. had significant morbidity with nonsignificant inhibition of tumor growth by virus alone, and no antitumor advantage of the CD/5FC system was seen by this route (data not shown). $10^{8}$ p.f.u. of VVCD (or control virus VVLuc, not shown) given i.p. had similar morbidity and a statistically significant inhibition 


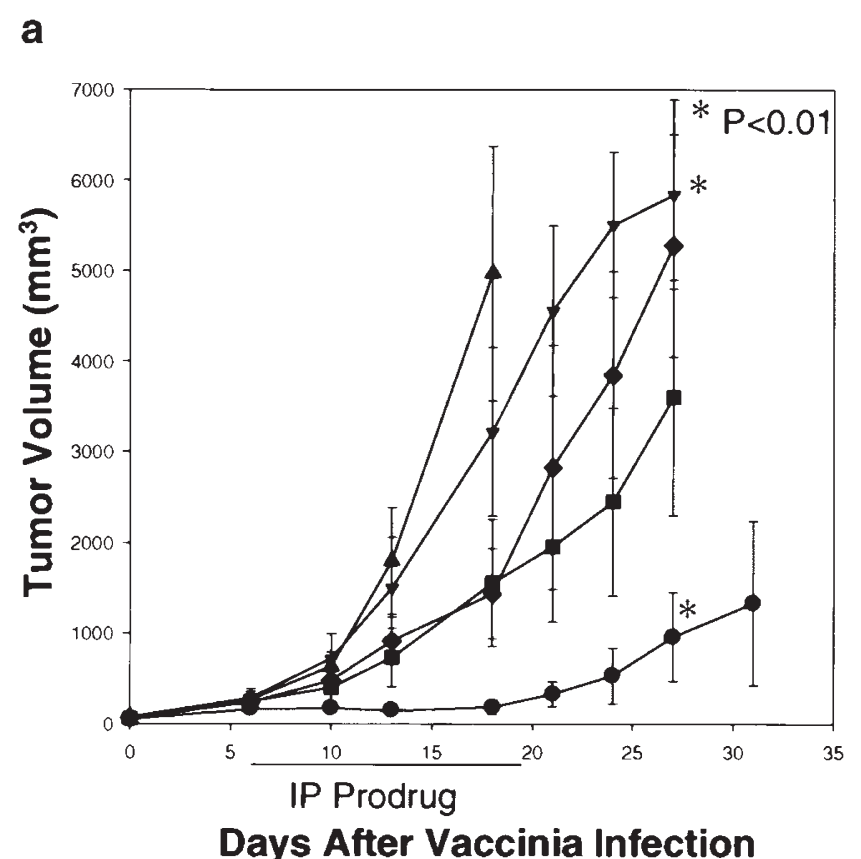

b

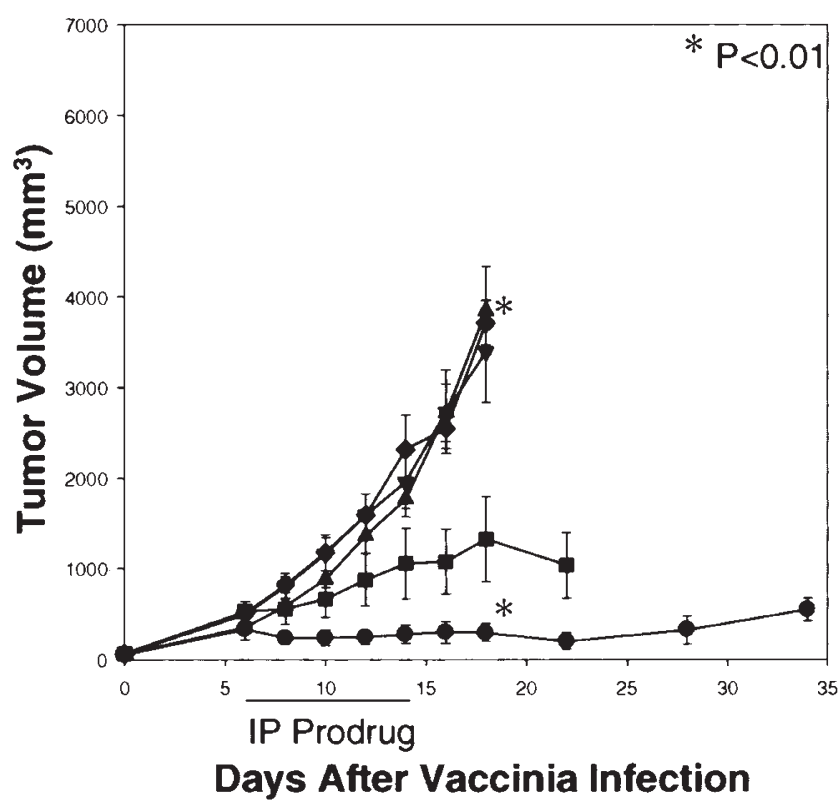

Figure 2 Mean tumor volumes \pm standard error after intratumoral treatment with $10^{7}\left(\right.$ a) or $10^{8}$ (b) p.f.u. of replicating vaccinia virus $(n=10)$. Mice were treated on day 0 when tumors were 50 to $100 \mathrm{~mm}^{3} .5 F C(\boldsymbol{\nabla})$, $5 F U(>)$, and PBS ( $\mathbf{\Delta})$ had no effect on tumor growth. VVCD alone ( $\mathbf{\square})$ showed some tumor inhibition that did not achieve statistical significance. ${ }^{*} V V C D+5 F C(\mathbf{O})$ had statistically significant inhibition of tumor growth compared with 5FC $(P<0.01)$ with $50 \%(a)$ and $20 \%(b)$ of mice achieving complete regressions.

of tumor growth (Figure 3) compared with PBS. There was no added antitumor effect from the addition of 5FC. This may reflect the rapid tumor cell killing by virus alone.

\section{In vivo viral pathogenicity}

Minimal toxicity was seen when $10^{7}$ p.f.u. of vaccinia was given intratumorally. At $10^{8}$ p.f.u. i.t. and both $10^{7}$ and

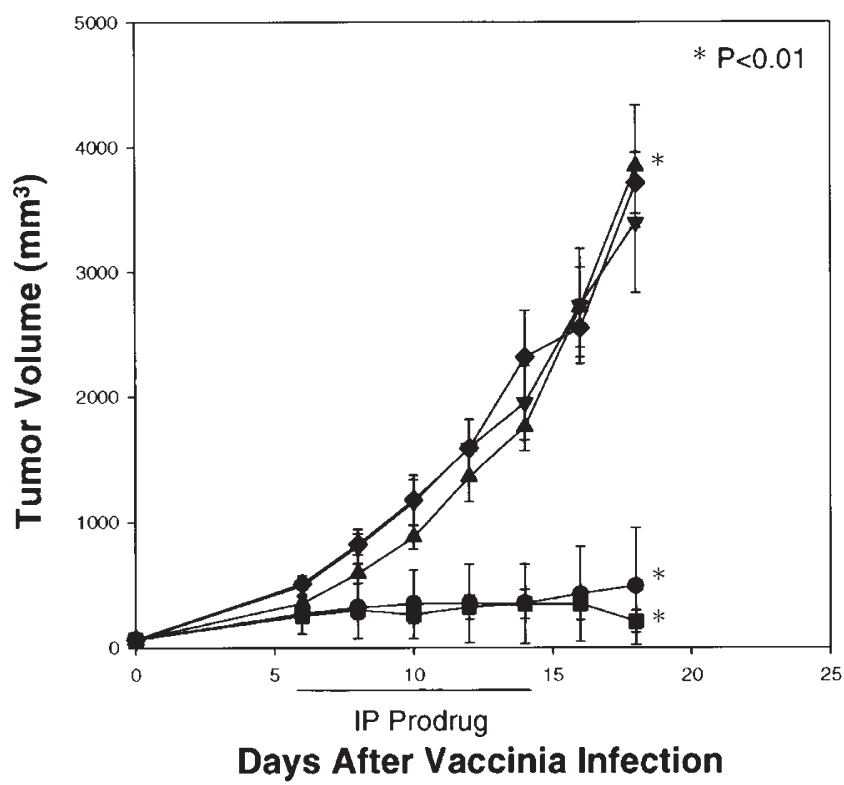

Figure 3 Mean tumor volume \pm standard error of the mean after intraperitoneal treatment with $10^{8}$ p.f.u. of replicating vaccinia virus $(n=10)$. $5 F C(\boldsymbol{\nabla}), 5 F U(\boldsymbol{\bullet})$, and PBS $(\boldsymbol{\Delta})$ had no effect on tumor growth. ${ }^{*}$ Both $V V C D$ alone $(\mathbf{\square})$ and $V V C D+5 F C(\bullet)$ had statistically significant inhibition of tumor growth compared to PBS $(P<0.01)$.

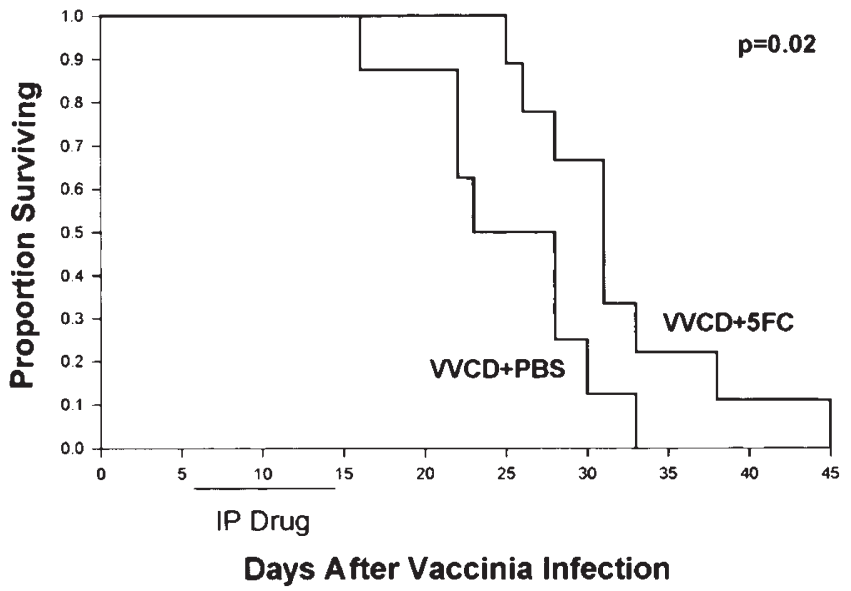

Figure 4 Survival of nude mice after treatment with $10^{8}$ p.f.u. of replicating VVCD + PBS or VVCD + 5FC intraperitoneally $(n=10)$. Death was virus-mediated as shown by weight loss, and skin lesions (pox). Tumors remained small as a result of treatment and were not the cause of death. The addition of 5FC significantly improved survival $(P=0.02)$ compared with PBS.

$10^{8}$ p.f.u. of vaccinia given systemically (i.p.), significant viral pathogenicity was seen in the form of weight loss, skin lesions and death. In the group that received $10^{8}$ p.f.u. i.p. VVCD and 5-FC there was a statistically significant prolongation of survival (Figure 4), presumably due to 5-FU (converted from 5-FC) mediated inhibition of viral replication, similar to that seen in vitro above. A similar improvement in survival was seen in the i.t. group as well. When the i.p. experiment was repeated with prolonged 5-FC treatment $(1180 \mathrm{mg} / \mathrm{kg}$ i.p. daily $\times 28$ days) there was no further survival advantage (data not shown).

In order to evaluate the cause of morbidity, mouse 
tissues were harvested and viral titers determined (Figure 5). As well as the high titers found in tumor and ovary, there were also relatively high titers in spleen, brain tissue, and bone marrow which could explain systemic pathogenicity. Minimal virus (less than 10 p.f.u./mg, not shown) was recovered from liver and no virus was recoverable from blood samples. An overall reduction of viral titers was seen in the group treated with 5-FC and this further highlights the effect that 5FU has on viral replication. Complete blood counts (CBC) were obtained to evaluate bone marrow pathology. Interestingly, bone marrow suppression was seen only in the group that received both VVCD and 5-FC (data not shown), and was likely 5-FU-mediated. Bone marrow suppression was not seen in the groups that got vaccinia alone, suggesting that this is an unlikely cause of vaccinia-mediated death.

\section{Discussion}

Tumor-directed gene therapy is limited by inefficient infectivity and low levels of gene expression in vivo from currently available viral vectors. The use of replicating viruses should overcome these limitations as they may allow for progressive infection of surrounding tumor cells and lead to a higher percentage of cells expressing the gene of interest. Currently, modified adenoviruses and herpes viruses are being investigated as replicating viruses for tumor-directed gene therapy. Adenoviruses have been modified to allow replication in p53- mutant tumor cells, which resulted in tumor selectivity and onco-

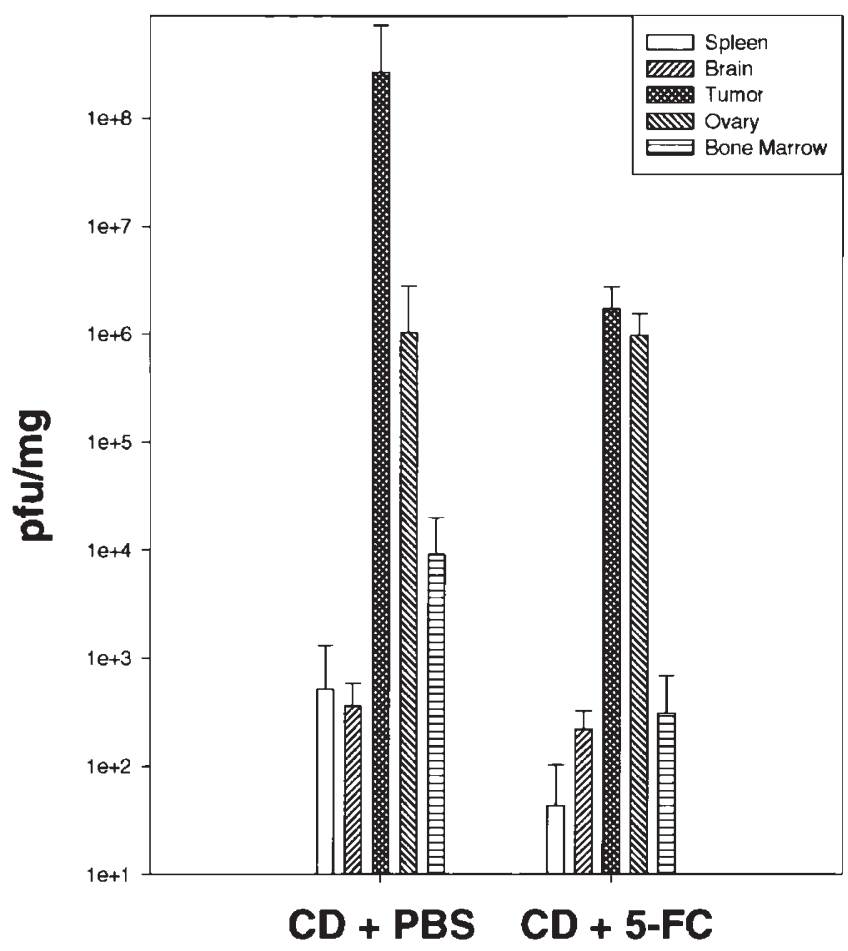

Figure 5 Viral titers (mean \pm standard deviation) of tissues 21 days after infection with VVCD + PBS or VVCD +5-FC. Nude mice $(n=3)$ were infected with $10^{7}$ p.f.u. VVCD i.p. and treated with PBS or 5-FC $(1080 \mathrm{mg} / \mathrm{kg}$ ) daily starting day 6. On day 21 after infection, tissues were harvested, homogenized and a standard plaque assay was performed. Differences between groups were not statistically significant. lysis. ${ }^{6,12,20}$ Replicating herpes viruses have been engineered to have tumor-specific oncolytic effects. . $8,11,21,22^{\text {Inac- }}$ tivation of the ribonucleotide reductase gene or the neurovirulence gene $(\gamma 34.5)$ of herpes virus allows tumor specificity with decreased neurotoxicity. ${ }^{7,11,22}$ While replication improves the efficiency of both adenoviruses and herpes viruses, neither has been shown to be efficient and specific enough to have antitumor effects as a systemically delivered vector.

In many ways a replicating vaccinia virus is superior to other replicating virus vectors. As a cytoplasmic virus nuclear localization is unnecessary, and since it supplies its own replication machinery there is minimal reliance on host factors. Vaccinia synthetic promoters lead to extremely high levels of gene expression. ${ }^{23-25}$ Vaccinia virus replicates efficiently in a wide variety of human tumors and can be produced in very high titers in Hela cell spinner cultures. Most important is our previous observation that vaccinia is tumor selective in vivo. ${ }^{26}$

In these experiments, we used a thymidine kinasedeleted vaccinia virus which has previously been shown to be less virulent than wild-type virus. ${ }^{17}$ We have shown higher gene expression in subcutaneous tumors after i.v., i.p. or i.t. delivery of a luciferase-expressing vaccinia virus compared with other tissues. Remarkably, peak tumor luciferase activity is similar between the three different routes of delivery. A $t k$-deleted vaccinia requires either host thymidine kinase (active in dividing cancer cells) or host nucleotides in order to synthesize DNA and replicate. This may be the mechanism of in vivo tumor selectivity demonstrated.

We studied the CD/5-FC enzyme/prodrug system because it is known to have a significant bystander effect. $^{27,28}$ It has been shown to have an antitumor effect when used in retroviral and adenoviral constructs, ${ }^{29-31}$ and when used systemically with vaccinia in a murine hepatic metastases model. ${ }^{18,32}$ The active metabolite 5-FU is a known inhibitor of DNA synthesis and may provide an antiviral effect through inhibition of viral replication.

We have shown that the interactions between a replicating vaccinia virus and enzyme/prodrug system are complex. In vitro, replicating vaccinia was cytotoxic with or without the addition of prodrug at higher MOIs $(>0.1)$, likely because of the speed with which vaccinia kills cells compared with the slow onset of activity of the enzyme prodrug system. At lower doses viral replication alone may not be enough to see an effect in rapidly dividing cells, but the bystander effect of the enzyme/prodrug system resulted in $40 \%$ cytotoxicity. Recoverable virus was decreased up to 300-fold by the addition of prodrug with no deleterious effects on cytotoxicity, indicating that it may be effective for rescue from virus-mediated pathogenicity.

In vivo, similar effects were seen. At high i.p. doses $\left(10^{8}\right.$ p.f.u.) there was significant inhibition of tumor growth with or without the addition of prodrug. There was a benefit to prodrug when virus $\left(10^{7}\right.$ or $10^{8}$ p.f.u. was given directly into the tumor resulting in $50 \%$ complete regressions and a dramatic inhibition of tumor growth overall. This may be a reflection of less viral dissemination throughout the tumor compared with systemic routes of injection leading to a necessary and effective bystander effect from the CD/5-FC system. As well, at lower doses of virus ( $10^{6}$ p.f.u.) delivered systemically in a model of liver metastases, no effect from virus repli- 
cation alone was observed, but prodrug delivery resulted in antitumor effects and prolongation of survival. ${ }^{32}$ In the current study, mice receiving $10^{7}$ or $10^{8}$ p.f.u. of vaccinia ultimately died from vaccinia pathogenicity in a dosedependent fashion. Survival of nude mice receiving $10^{8}$ p.f.u. of systemic vaccinia was significantly prolonged in the presence of prodrug, suggesting an antiviral effect of the converted prodrug. Although we found no evidence for bone marrow toxicity, other factors may be the cause of death in these animals. Interestingly, histological examination performed on infected tissues showed only tumor necrosis, ovarian pathology and proliferative hepatic changes with no evidence of central nervous system effects (unpublished results). It is difficult therefore to define the specific cause of death in this model and this is being studied further.

The combination of a tumor-selective replicating vaccinia virus and an enzyme/prodrug system may be a potent vector for cancer gene therapy. ${ }^{10,11,13,33} \mathrm{~A}$ replicating virus is capable of directly causing tumor cell death, but it is ultimately limited by the immune system. An enzyme-prodrug system, delivered by a replicating virus could enhance the tumor response through its bystander effects. Converted prodrugs which inhibit DNA synthesis however, theoretically inhibit viral replication in a similar manner. This may be a disadvantage if it limits virus-mediated tumor cell death, however an enzyme/prodrug system which inhibits viral DNA synthesis may act as a safety valve to rescue the host from virus-mediated pathogenicity.

We have shown that systemic injection of an oncolytic replicating vaccinia virus leads to high gene expression within tumor cells in vivo and results in a significant antitumor effect. Despite the use of a thymidine kinasedeleted virus and an enzyme/prodrug system, significant pathogenicity was seen in nude mice. Although this is negligible in immunocompetent mice, future clinical trials with a systemically delivered, replicating virus will need to be safe from pathogenicity. Currently we are working at improving the safety profile of these viruses further by mutating other genes and expressing more potent enzyme/prodrug systems. The efficiency, high gene expression, and tumor selectivity of this replicating vaccinia virus make it worthy of continued investigation for tumor-directed gene therapy.

\section{Materials and methods}

\section{Cell lines}

MC38, a non-metastatic colon adenocarcinoma cell line from C57BL/6 mice is used extensively in our branch. The human colon adenocarcinoma cell lines WiDr, HT29 and H508, cervical adenocarcinoma (Hela S3) and monkey kidney fibroblasts (CV1) were obtained from the American Type Culture Collection (ATCC, Manassass, VA, USA). All cell lines were grown in Dulbecco's modified Eagle's medium (DMEM-10) supplemented with $10 \%$ heat-inactivated fetal calf serum, $2 \mathrm{~mm}$ glutamine, $1 \%$ penicillin/streptomycin (10 000 units $/ \mathrm{ml}$ ), and $0.2 \%$ fungizone $(250 \mu \mathrm{g} / \mathrm{ml})$ (all Biofluids, Rockville, MD, USA). Cell lines were maintained in an incubator at $37^{\circ} \mathrm{C}$ with $5 \% \mathrm{CO}_{2}$, and serially passaged every 3 to 4 days. Hela cells in suspension culture were grown in 3L spinner flasks (Bellco Glass, Vineland, NJ, USA) at $37^{\circ} \mathrm{C}$ with no $\mathrm{CO}_{2}$.
Vaccinia viruses

The recombinant vaccinia viruses VVLuc (expressing the firefly luciferase gene) and VVCD (expressing the E. coli cytosine deaminase gene) were developed in our laboratory and have been described previously. ${ }^{18,26}$ Recombinant vaccinia viruses were amplified in Hela cell spinner cultures for 3 days, purified by ultracentrifugation over a sucrose cushion, and titered on confluent CV1 cells. ${ }^{34}$

\section{MTT assays}

On day $-2,5 \times 10^{3}$ cells per well were plated in a 96-well plate $\left(5 \times 10^{5}\right.$ cells per well in six-well plates for replicating vaccinia) and incubated overnight in $5 \% \mathrm{CO}_{2}$ at $37^{\circ} \mathrm{C}$. The next day, cells were infected with vaccinia virus at varying MOIs. 5-Fluorocytosine (5-FC, $1 \mathrm{~mm}$, Sigma Chemical, St Louis MO, USA) or control was added on day 0 . MTT assays were performed on days 1, 3 and 5 as previously described. ${ }^{19}$ All assays were performed in triplicate. Cell viability was expressed relative to control cells grown in media alone.

\section{Viral titers}

On days 3 and 5 after viral infection cell lysates were sampled for virus titers. Cells were harvested in $1 \mathrm{ml}$ of DMEM supplemented with $2.5 \%$ serum and subjected to one freeze-thaw cycle. $500 \mu \mathrm{l}$ of cell lysate was used in a standard plaque assay. ${ }^{34}$

\section{Mice}

Six-week-old female, athymic (C57BL/6) mice were obtained from the National Institutes of Health small animal facility (Frederick, MD, USA). They were housed in standard conditions and given food and water ad libitum. All animal studies were approved by the Animal Care and Use Subcommittee of the Animal Sciences Branch, National Cancer Institute.

\section{Tumor model}

$10^{5}$ MC38 cells in $100 \mu$ l of DMEM were injected into the right flank of each mouse and allowed to grow for 1014 days. When the subcutaneous tumors reached a volume of 50 to $100 \mathrm{~mm}^{3}$, vaccinia virus (VVLuc or VVCD) was injected intratumorally (i.t.), intraperitoneally (i.p.), or intravenously (i.v.).

\section{Prodrug}

5-Fluorocytosine (5-FC, Sigma Chemical) was used at $1 \mathrm{~mm}$ in phosphate buffered saline (PBS, pH 7.4, Biofluids) for the in vitro studies. It was dissolved in $2 \mathrm{ml}$ DMEM per mouse ( $1180 \mathrm{mg} / \mathrm{kg} /$ day) for the in vivo studies. 5-Fluorouracil (5-FU, Sigma Chemical) was used at $1 \mathrm{~mm}$ in vitro and $30 \mathrm{mg} / \mathrm{kg} /$ day in vivo.

\section{In vivo marker study}

On days 2, 4, 6, 8, 10 and 14 following i.t., i.p., or i.v. injection of VVLuc, three mice per group were killed, and 50 to $100 \mathrm{mg}$ samples of liver, spleen, lung, heart, kidney, small bowel, skin, ovary and tumor were removed and homogenized in $750 \mu \mathrm{l}$ of reporter lysis buffer (Promega, Madison, WI, USA) and kept at $-70^{\circ} \mathrm{C}$ until assayed. The luciferase assay was done according to the protocol (Luciferase Assay System, Promega). In brief, $20 \mu \mathrm{l}$ of thawed homogenate was added to $100 \mu \mathrm{l}$ of room temperature luciferase assay reagent, and the reaction read 
for $10 \mathrm{~s}$ in a luminometer (Turner Designs, Sunnyvale, CA, USA). Each sample was done in duplicate.

Total protein was assayed using the BCA Protein Assay Reagent Kit (Pierce, Rockford, IL, USA) using the Microtiter Plate Protocol. $200 \mu$ l of working reagent was added to $10 \mu \mathrm{l}$ of each of the thawed tissue homogenates (above) in a 96-well plate. The plate was shaken for $30 \mathrm{~s}$ and incubated at $37^{\circ} \mathrm{C}$ for $1 \mathrm{~h}$. After cooling to room temperature, the absorbance was measured at $570 \mathrm{~nm}$. Known concentrations of bovine serum albumin (BSA set, Pierce) were measured to obtain a standard curve. The luciferase assay results were reported as relative light units (RLU) per minute per milligram total protein.

\section{In vivo cytotoxicity}

VVCD, VVLuc or HBSS (Hank's balanced salt solution; Biofluids) control was injected intratumorally (in $100 \mu \mathrm{l}$ of HBSS/0.1\% BSA, Calbiochem, La Jolla, CA, USA) or intraperitoneally (in $2 \mathrm{ml}$ of HBSS/0.1\% BSA) when the tumors were 50 to $100 \mathrm{~mm}^{3}$. Starting day 6 following viral injection, $2 \mathrm{ml}$ of 5-FC (1180 mg/ kg), 5-FU (30 mg/kg, Sigma Chemical) or PBS was injected i.p. daily for 9, 14 or 28 days. Tumor width and length was measured twice weekly by a blinded investigator. Tumor volume was calculated as $\left((\text { width })^{2} \times\right.$ length $) 0.52 .{ }^{35}$

\section{Viral pathogenicity}

Viral pathogenicity was assessed with complete blood counts and determinations of viral titers in various organs, 8 and 15 days after injection of VVCD. $250 \mu l$ of blood was aspirated into mini-collection tubes (Becton Dickinson, Fairland Lakes, NJ, USA) for complete blood counts. For viral titer determinations, 50 to $100 \mathrm{mg}$ samples of liver, spleen, brain, tumor, ovary, bone marrow and blood were homogenized in HBSS (without BSA) and kept at $-70^{\circ} \mathrm{C}$ until use. $500 \mu$ l of the homogenate was incubated on CV1 cells at $37^{\circ} \mathrm{C}$ in $5 \% \mathrm{CO}_{2}$ and titers determined as above. Viral titers were standardized to total protein.

\section{Statistics}

Data were presented as mean \pm standard deviation and the Mann-Whitney test was used when appropriate. Tumor volumes between groups were assessed using the Kruskal-Wallis nonparametric analysis of variance and Dunn's multiple comparisons test. Survival analysis was performed using the method of Kaplan-Meier, ${ }^{36}$ and differences between curves were assessed using the log-rank test. ${ }^{37}$

\section{Acknowledgements}

The authors thank Drs Charles Brown, Peter Wu and James Huang for their assistance with this work.

\section{References}

1 Siders WM, Halloran PJ, Fenton RG. Transcriptional targeting of recombinant adenoviruses to human and murine melanoma cells. Cancer Res 1996; 56: 5638-5646.

2 Parr $\mathrm{MJ}$ et al. Tumor-selective transgene expression in vivo mediated by an E2F-responsive adenoviral vector. Nature Med 1997; 3: 1145-1149.

3 Dmitriev I et al. An adenovirus vector with genetically modified fibers demonstrates expanded tropism via utilization of a coxsackievirus and adenovirus receptor-independent cell entry mechanism. J Virol 1998; 72: 9706-9713.

4 Park BJ et al. Augmentation of melanoma-specific gene expression using a tandem melanocyte-specific enhancer results in increased cytotoxicity of the purine nucleoside phosphorylase gene in melanoma. Hum Gene Ther 1999; 10: 889-898.

5 Martuza RL et al. Experimental therapy of human glioma by means of a genetically engineered virus mutant. Science 1991; 252: 854-856.

6 Heise C et al. ONYX-015, an E1B gene-attenuated adenovirus, causes tumor-specific cytolysis and antitumoral efficacy that can be augmented by standard chemotherapeutic agents. Nature Med 1997; 3: 639-645.

7 Advani SJ et al. Replication-competent, nonneuroinvasive genetically engineered herpes virus is highly effective in the treatment of therapy-resistant experimental human tumors. Cancer Res 1999; 59: 2055-2058.

8 Walker JR et al. Local and systemic therapy of human prostate adenocarcinoma with the conditionally replicating herpes simplex virus vector G207. Hum Gene Ther 1999; 10: 2237-2243.

9 Lane DP. Killing tumor cells with viruses - a question of specificity. Nature Med 1998; 4: 1012-1013.

10 Miyatake S-I, Martuza RL, Rabkin SD. Defective herpes simplex virus vectors expressing thymidine kinase for the treatment of malignant glioma. Cancer Gene Ther 1997; 4: 222-228.

11 Chase M, Chung RY, Chiocca EA. An oncolytic viral mutant that delivers the CYP2B1 transgene and augments cyclophosphamide chemotherapy. Nature Biotech 1998; 16: 444-448.

12 Wildner O, Blaese RM, Morris JC. Therapy of colon cancer with oncolytic adenovirus is enhanced by the addition of herpes simplex virus-thymidine kinase. Cancer Res 1999; 59: 410-413.

13 Aghi $\mathrm{M}$ et al. Multimodal cancer treatment mediated by a replicating oncolytic virus that delivers the oxazaphosphorine/rat cytochrome P450 2B1 and ganciclovir-herpes simplex virus thymidine kinase gene therapies. Cancer Res 1999; 59: 3861-3865.

14 Carroll MW, Moss B. Poxviruses as expression vectors. Curr Opin Biotechnol 1997; 8: 573-577.

15 Peplinski GR, Tsung K, Norton JA. Vaccinia virus for human gene therapy. Surg Oncol Clin North Am 1998; 7: 575-588.

16 Moss B. Genetically engineered poxviruses for recombinant gene expression, vaccination, and safety. Proc Natl Acad Sci USA 1996; 93: 11341-11348.

17 Buller RM et al. Decreased virulence of recombinant vaccinia virus expression vectors is associated with a thymidine kinasenegative phenotype. Nature 1985; 317: 813-815.

18 Gnant MFX, Puhlmann M, Alexander HR Jr, Bartlett DL. Systemic administration of a recombinant vaccinia virus expressing the cytosine deaminase gene and subsequent treatment with 5fluorocytosine leads to tumor specific gene expression and prolongation of survival in mice. Cancer Res 1999; 59: 3396-3404.

19 Puhlmann $\mathrm{M}$ et al. Thymidine kinase deleted vaccinia virus expressing purine nucleoside phosphorylase as a vector for tumor directed gene therapy. Hum Gene Ther 1999; 10: 649-657.

20 Bischoff JR et al. An adenovirus mutant that replicates selectively in p53-deficient human tumor cells (see comments). Science 1996; 274: 373-376.

21 Nicolo M, Chiocca EA. Marker gene transfer and oncolysis of human Y79 retinoblastoma cells mediated by herpes simplex virus mutants. Ophthal Surg 1998; 30: 30-36.

22 Andreansky $S$ et al. Evaluation of genetically engineered herpes simplex viruses as oncolytic agents for human malignant brain tumors. Cancer Res 1997; 57: 1502-1509.

23 Chakrabarti S, Sisler JR, Moss B. Compact, synthetic, vaccinia virus early/late promoter for protein expression. BioTechniques 1997; 23: 1094-1097.

24 Davison AJ, Moss B. Structure of vaccinia virus late promoters. J Mol Biol 1989; 210: 771-784.

25 Davison AJ, Moss B. Structure of vaccinia virus early promoters. J Mol Biol 1989; 210: 749-769.

26 Puhlmann $\mathrm{M}$ et al. Vaccinia as a vector for tumor directed gene therapy: biodistribution of a thymidine kinase deleted mutant. Cancer Gene Ther 2000; 7: 66-73. 
27 Trinh QT et al. Enzyme-prodrug gene therapy: comparison of cytosine deaminase/5-fluorocytosine versus thymidine kinaseganciclovir enzyme/prodrug systems in a human colorectal carcinoma cell line. Cancer Res 1995; 55: 4808-4812.

28 Huber BE et al. Metabolism of 5-fluorocytosine to 5-fluorouracil in human colorectal tumor cells transduced with the cytosine deaminase gene: significant antitumor effects when only a small percentage of tumor cells express cytosine deaminase. Proc Natl Acad Sci USA 1994; 91: 8302-8306.

29 Hirschowitz EA et al. In vivo adenovirus-mediated gene transfer of the Escherichia coli cytosine deaminase gene to human colon carcinoma-derived tumors induces chemosensitivity to 5-fluorocytosine. Hum Gene Ther 1995; 6: 1055-1063.

30 Ohwada A, Hirschowitz EA, Crystal RG. Regional delivery of an adenovirus vector containing the Escherichia coli cytosine deaminase gene to provide local activation of 5-fluorocytosine to suppress the growth of colon carcinoma metastatic to liver. Hum Gene Ther 1996; 7: 1567-1576.

31 Topf N, Worgall S, Hackett NR, Crystal RG. Regional 'pro-drug' gene therapy: intravenous administration of an adenoviral vec- tor expressing the E. coli cytosine deaminase gene and systemic administration of 5-fluorocytosine suppresses growth of hepatic metastasis of colon carcinoma. Gene Therapy 1998; 5: 507-513.

32 Gnant M, Puhlmann M, Bartlett DL, Alexander HR. Regional versus systemic delivery of recombinant vaccinia virus as suicide gene therapy for murine liver metastases. Ann Surg 1999; 230: 352-361.

33 Huber BE, Richards CA, Austin EA. Virus-directed enzyme/ prodrug therapy (VDEPT). Ann NY Acad Sci 1994; 716: 104-114.

34 Earl PL, Moss B. Expression of proteins in mammalian cells using vaccinia viral vectors. In: Ausubel FM et al (eds). Current Protocols in Molecular Biology. John Wiley: New York, 1998, pp 16.15.1-16.18.11.

35 O'Reilly MS et al. Endostatin: an endogenous inhibitor of angiogenesis and tumor growth. Cell 1997; 88: 277-285.

36 Kaplan EL, Meier P. Non-parametric estimation from incomplete observation. J Am Stat Assoc 1958; 53: 457-481.

37 Mantel N. Evaluation of survival data and two new rank order statistics arising in its consideration. Cancer Chem Rep 1966; 50: 163-170. 